\title{
Relativistic 2-body Bottomonium decays
}

\author{
Andrea Barducci ${ }^{1,2, a}$, Riccardo Giachetti ${ }^{1,2, b}$, and Emanuele Sorace ${ }^{2, c}$ \\ ${ }^{1}$ Physics Department, University of Firenze, Via G. Sansone 1, 50019 Sesto Fiorentino, Italy. \\ ${ }^{2}$ Istituto Nazionale Fisica Nucleare, Sezione di Firenze, Via G. Sansone 1, 50019 Sesto Fiorentino, Italy
}

\begin{abstract}
We calculate some radiative decays of Bottomonium in a covariant scheme for two interacting fermions. We present their branching ratios and their absolute widths. A comparison with experimental data shows a good agreement with our results. Some decays for which data are not available are compared with other theoretical previsions.
\end{abstract}

\section{Introduction}

In some previous papers we have developed a completely covariant framework for treating bound systems of two relativistic fermions of arbitrary masses [1-4]. The main benefits of our approach are that the relativistic contributions are completely taken into account. In particular the corrections due to the two spin-orbit couplings, to the reduced mass and to the recoil, necessary when the starting framework is non-relativistic, are included since the beginning. In that scheme we calculated the lowest levels and some Hyperfine splittings of Hydrogenic atoms [1, 2, 4]. In [3] we presented the results of the evaluation of meson masses, using the Cornell potential with the addition of a Breit term correction. Notice that the vector Coulomb-like term of the Cornell potential is correctly coupled to the energy, whereas the linear scalar term is coupled to the mass in order to ensure the confinement [5]. We found a very good agreement for the heaviest families of mesons $(b \bar{b}, c \bar{c}, s \bar{s}, B c, B s, D s)$ and we proved that the same method was able to reproduce with good accuracy the masses of the light mesons $u \bar{d}$ also. The quark masses, the string tension and $\alpha_{S}$ constants were fitted independently for each family: it is remarkable that the fits gave back the same values for the string tension at least for the heavier families, in agreement with the flavor independence predicted by the theory. Moreover we found that the fitted values of the running constant $\alpha_{S}$ closely follows the QCD behavior up to a multiplicative factor. The calculations proved the fundamental contribution of the Breit term in order to recover the correct masses. In [4] we presented the electromagnetic coupling to atoms and we calculated the widths of some hyperfine decays. The same coupling has been used in order to investigate the electromagnetic decays of bottomonium [6]. These will be presented in the following sections. We refer to [1-6] for details and for the comparison of our methods with others quoted in an extended bibliography.

\footnotetext{
ae-mail: barducci@fi.infn.it

be-mail: giachetti@fi.infn.it

ce-mail: sorace@fi.infn.it
} 


\section{The interacting two-fermion problem}

The equation for the two fermions of masses $m_{1}, m_{2}$ and eigenvalue $\lambda$ reads:

$$
\begin{gathered}
{\left[\left(\gamma_{(1)}^{0} \gamma_{(1)_{a}}-\gamma_{(2)}^{0} \gamma_{(2) a}\right) q_{a}+\right.} \\
\frac{1}{2}\left(\gamma_{(1)}^{0}+\gamma_{(2)}^{0}\right)\left(m_{1}+m_{2}+\sigma r\right)+\frac{1}{2}\left(\gamma_{(1)}^{0}-\gamma_{(2)}^{0}\right)\left(\left|m_{1}-m_{2}\right|\right) \\
\left.-\left(\lambda+\frac{b}{r}\right)+V_{B}(r)\right] \Psi(\boldsymbol{r})=0 .
\end{gathered}
$$

In (1) $\Psi(\vec{r})$ is a 16-component spinor obtained by reordering the tensor product of the two quarks so as to collect singlets and triplets for the different eigenvalues of the mass, $\vec{r}$ and $\vec{q}$ are the Wigner relative coordinate and momentum [1,4]. Moreover: $\gamma_{(i)}^{0}, \gamma_{(i)}$ are the $\gamma$ matrices acting on the space of the two fermions; $b$ is the fine structure constant $\alpha$ and $\sigma=0$ in the atomic case; the vector and scalar parts of the Cornell potential respectively give the $(\lambda+b / r)$ and $\left(m_{1}+m_{2}+\sigma r\right)$ terms where $b=(4 / 3) \alpha_{Q C D}$ and $\sigma$ is the string tension. Finally the Breit potential $V_{B}(r)$ has the form

$$
V_{B}(r)=\frac{\tilde{b}}{2 r} \gamma_{(1)}^{0} \gamma_{(1) a} \gamma_{(2)}^{0} \gamma_{(2)_{b}}\left(\delta_{a b}+\frac{r_{a} r_{b}}{r^{2}}\right)
$$

with $\tilde{b}=b$ for mesons and $\tilde{b}=\kappa_{1} \kappa_{2} \alpha$ for atoms, where $\kappa_{1}, \kappa_{2}$ are the anomalous multiplicative corrections to the fermion magnetic moments.

The results for atomic HFS are reported in Tables 1 and 2.

Table 1. The 1s, $2 \mathrm{~s}$ HFS, $\Delta_{\mathrm{HFS}}(1 s), \Delta_{\mathrm{HFS}}(2 s)$ (second and fourth columns) and the corresponding experimental values (third and fifth columns) for some Hydrogenic atoms indicated, in the first column, by their two components. $\mathrm{p}$ is the proton, $\mathrm{e}$ is the electron, $\mu^{+}, \mu$ the positive and negative $\mu$ mesons, ${ }^{3} \mathrm{He}^{+}$, the Helium 3 ion. According to a common use, we give the results of the first three lines in $\mathrm{MHz}$ and those of the last two in meV.

\begin{tabular}{lrrrr}
\hline Atom & \multicolumn{2}{c}{$\Delta_{\mathrm{HFS}}(1 s)$} & \multicolumn{2}{c}{$\Delta_{\mathrm{HFS}}(2 s)$} \\
\hline$(\mathrm{p}, \mathrm{e})$ & 1420.595 & 1420.405 & 177.580 & 177.557 \\
$\left(\mu^{+}, \mathrm{e}\right)$ & 4464.481 & 4463.302 & 558.078 & 558. \\
$\left({ }^{3} \mathrm{He}^{+}, \mathrm{e}\right)$ & -8665.637 & -8665.650 & -1083.347 & -1083.355 \\
\hline$(\mathrm{p}, \mu)$ & 182.621 & 182.638 & 22.828 & 22.815 \\
$\left({ }^{3} \mathrm{He}^{+}, \mu\right)$ & -1372.194 & -1334.730 & -171.544 & -166.645 \\
\hline
\end{tabular}

The masses for the lowest Bottomonium states are given in Table 3. They have been obtained from eq. (1) by fitting the Bottom mass, $\sigma$ and $b$.

\section{Decay line widths}

We retrace the general quantum mechanical procedure to calculate the transition probability between the hyperfine split $s$-states. We consider in the Coulomb gauge the wave function of a photon with momentum $k$ and polarization $\boldsymbol{\epsilon}_{\sigma}$ :

$$
\boldsymbol{A}_{(k, \sigma)}(x)=\frac{\sqrt{4 \pi}}{\sqrt{2 \omega}} \boldsymbol{\epsilon}_{\sigma} e^{-i k x} .
$$


Table 2. The same as Table 1 for the HFS splittings $\Delta_{\mathrm{HFS}}\left(2 p^{1 / 2}\right)$ and $\Delta_{\mathrm{HFS}}\left(2 p^{3 / 2}\right)$. Again we give the results of the first three lines in $\mathrm{MHz}$ and those of the last two in meV.

\begin{tabular}{lrrrr}
\hline Atom & \multicolumn{2}{c}{$\Delta_{\mathrm{HFS}}\left(2 p^{1 / 2}\right)$} & \multicolumn{2}{c}{$\Delta_{\mathrm{HFS}}\left(2 p^{3 / 2}\right)$} \\
\hline$(\mathrm{p}, \mathrm{e})$ & 59.196 & 59.221 & 23.678 & 24.0 \\
$\left(\mu^{+}, \mathrm{e}\right)$ & 186.252 & 187.0 & 74.629 & 74.0 \\
$\left({ }^{3} \mathrm{He}^{+}, \mathrm{e}\right)$ & -361.100 & - & -144.385 & - \\
\hline$(\mathrm{p}, \mu)$ & 76.82 & 78.20 & 31.15 & 32.48 \\
$\left({ }^{3} \mathrm{He}^{+}, \mu\right)$ & -57.028 & 58.713 & -22.700 & -24.291 \\
\hline
\end{tabular}

Table 3. The $b \bar{b}$ levels in MeV. First column: term symbol, $I^{G}\left(J^{P C}\right)$ numbers , particle name. $\sigma=1.111 \mathrm{GeV} / \mathrm{fm}$, $\alpha=0.3272, m_{b}=4725.5 \mathrm{MeV}$. Experimental data from [7]. Data by different approaches are in [8]. ${ }^{(*)}$ see [9].

\begin{tabular}{lcc}
\hline State & Experimental & Numerical \\
\hline$\left(1^{1} s_{0}\right) 0^{+}\left(0^{-+}\right) \eta_{b}$ & $9398.0 \pm 3.2$ & 9390.39 \\
$\left(1^{3} s_{1}\right) 0^{-}\left(1^{--}\right) \Upsilon$ & $9460.30 \pm .25$ & 9466.10 \\
$\left(1^{3} p_{0}\right) 0^{+}\left(0^{++}\right) \chi_{b 0}$ & $9859.44 \pm .73$ & 9857.41 \\
$\left(1^{3} p_{1}\right) 0^{+}\left(1^{++}\right) \chi_{b 1}$ & $9892.78 \pm .57$ & 9886.70 \\
$\left(1^{1} p_{1}\right) 0^{-}\left(1^{+-}\right) h_{b}$ & $9898.60 \pm 1.4$ & 9895.35 \\
$\left(1^{3} p_{2}\right) 0^{+}\left(2^{++}\right) \chi_{b 2}$ & $9912.21 \pm .57$ & 9908.14 \\
$\left(2^{1} s_{0}\right) 0^{+}\left(0^{-+}\right) \eta_{b}$ & $9974.0 \pm 4.4(*)$ & 9971.14 \\
$\left(2^{3} s_{1}\right) 0^{-}\left(1^{--}\right) \Upsilon$ & $10023.26 \pm .0003$ & 10009.04 \\
$\left(2^{3} p_{0}\right) 0^{+}\left(0^{++}\right) \chi_{b 0}$ & $10232.50 \pm .0009$ & 10232.36 \\
$\left(2^{3} p_{1}\right) 0^{+}\left(1^{++}\right) \chi_{b 1}$ & $10255.46 \pm .0005$ & 10256.58 \\
$\left(2^{1} p_{1}\right) 0^{-}\left(1^{+-}\right) h_{b}$ & $10259.8 \pm 1.6$ & 10263.62 \\
$\left(2^{3} p_{2}\right) 0^{+}\left(2^{++}\right) \chi_{b 2}$ & $10268.65 \pm .0007$ & 10274.26 \\
$\left(3^{3} s_{1}\right) 0^{-}\left(1^{--}\right) \Upsilon$ & $10355.20 \pm .0005$ & 10364.52 \\
\hline
\end{tabular}

Table 4. The Breit corrections in $\mathrm{MeV}$ for the lowest states.

\begin{tabular}{cccccc}
\hline$\eta_{b}(1 s)$ & $\Upsilon_{b}(1 s)$ & $\chi_{b 0}(1 p)$ & $\chi_{b 1}(1 p)$ & $h_{b}(1 p)$ & $\chi_{b 2}(1 p)$ \\
\hline-92.13 & -18.09 & -44.3 & -19.98 & -15.95 & -7.51 \\
\hline
\end{tabular}

The interaction Hamiltonian reads

$$
H_{\mathrm{int}}=q\left(z \boldsymbol{\alpha}_{(1)} \cdot \boldsymbol{A}^{(1)}-\boldsymbol{\alpha}_{(2)} \cdot \boldsymbol{A}^{(2)}\right)
$$

where $\boldsymbol{A}^{(1,2)}=\boldsymbol{A}\left(x_{(1,2)}\right), \boldsymbol{\alpha}_{(1,2)}=\gamma_{0(1,2)} \gamma_{(1,2)}$ are the Dirac $\boldsymbol{\alpha}$-matrices acting in the spinor space of the corresponding fermion $q=e$ for atoms, $q=2 / 3 e$ for Bottomonium and $z$ is the atomic number of the heavier fermion for Hydrogenic systems. We choose the frame with initial total momentum $\boldsymbol{P}_{i}=0$. 
We call $\psi_{i}(\boldsymbol{r})$ and $\psi_{f}(\boldsymbol{r})$ the 16-component spinors at initial and final energies, angular momenta and parities obtained after the factorization of the exponential corresponding to the wave function of the total momentum. Letting $\Delta=\left|m_{1}^{2}-m_{2}^{2}\right| /\left(2 \lambda_{i}^{2}\right), \lambda_{i}$ being the eigenvalue of the initial state, some straightforward calculations lead to the first perturbation order of the $S$-matrix element

$$
S_{f i}=-\frac{(2 \pi)^{4} i e}{\sqrt{2 \omega V}} \delta^{4}\left(P_{f}+k-P_{i}\right) \int d^{3} \boldsymbol{r} \frac{\sqrt{4 \pi}}{\sqrt{V^{2}}} \psi_{f}^{*}(\boldsymbol{r}) \boldsymbol{\epsilon}_{\sigma}^{*} \cdot\left[\widetilde{\boldsymbol{\alpha}}_{(1)} e^{-i\left(\frac{1}{2}-\Delta\right) \boldsymbol{k} \boldsymbol{r}}-\widetilde{\boldsymbol{\alpha}}_{(2)} e^{i\left(\frac{1}{2}+\Delta\right) \boldsymbol{k} \boldsymbol{r}}\right] \psi_{i}(\boldsymbol{r}) .
$$

Here $\widetilde{\boldsymbol{\alpha}}_{(j)}$ are the matrices obtained from $\boldsymbol{\alpha}_{(j)}$ by applying the unitary transformation generated by the change of basis we have made so to give the spinor components a convenient order chosen in terms of mass and angular momentum $[1,2]$. The $\delta^{4}$-function gives the energy-momentum conservation,

$$
P_{i}^{0}=P_{f}^{0}+\omega, \quad \boldsymbol{P}_{i}=\boldsymbol{P}_{f}+\boldsymbol{k}
$$

and contains the recoil due to the radiation emission.

For atomic systems we use the dipole approximation. Moreover in the initial and final wave functions entering the matrix element $S_{f i}$ the correction due to the spin-spin interactions due to the Breit term has been considered at the first perturbation order. We get:

$$
w=\frac{4}{3} \frac{\omega^{3}}{\hbar c^{3}} \Lambda_{f i}^{2}\left|\mu_{f i}\right|^{2}
$$

where

$$
\hbar \omega=\frac{\lambda_{i}+\lambda_{f}}{2 \lambda_{i}}\left(\lambda_{i}-\lambda_{f}\right), \quad \Lambda_{f i}^{2}=\frac{\lambda_{i}^{2}+\lambda_{f}^{2}}{2 \lambda_{i}^{2}},
$$

$\left|\mu_{f i}\right|^{2}$ is the common value of $\left|\boldsymbol{\epsilon}_{\sigma}^{*} \cdot \boldsymbol{d}_{f i}\right|^{2}$ for each of the two independent circular polarizations $\left(\epsilon_{1} \pm i \epsilon_{2}\right) / \sqrt{2}$ and

$$
\boldsymbol{d}_{f i}=-i e \int d^{3} \boldsymbol{r}\left(\frac{\boldsymbol{k}}{\omega} \cdot \boldsymbol{r}\right) \psi_{f}^{*}(\boldsymbol{r})\left[\widetilde{\boldsymbol{\alpha}}_{(1)}\left(\frac{1}{2}-\frac{m_{1}^{2}-m_{2}^{2}}{2 \lambda_{i}^{2}}\right)+\widetilde{\boldsymbol{\alpha}}_{(2)}\left(\frac{1}{2}+\frac{m_{1}^{2}-m_{2}^{2}}{2 \lambda_{i}^{2}}\right)\right] \psi_{i}(\boldsymbol{r}) .
$$

The widths for the HF Hydrogen decays are:

$$
\begin{array}{ll}
n=1 & \mathcal{P}_{H F S_{1}}=2.86610^{-15} s^{-1} \\
n=2 & \mathcal{P}_{H F S_{2}}=1.87110^{-15} s^{-1}
\end{array}
$$

approximately known

not found in literature

For the first levels of the muonic Hydrogen:

$$
n=1 \quad \mathcal{P}_{H F S_{1}}=2.49610^{-6} s^{-1} \quad \text { not found in literature }
$$

Contrary to the atomic cases, a rigorous perturbation expansion in the Breit term is not feasible for meson radiative decays. Indeed a remnant of the Breit correction is already present at the lowest order by means of the parameters entering the wave equation and its solutions. The $S$-matrix element is

$$
S_{f i}=-i e_{b} \frac{(2 \pi)^{4}}{\sqrt{2 \omega V}} \frac{\sqrt{4 \pi}}{\sqrt{V^{2}}} \delta^{4}\left(P_{f}+k-P_{i}\right)\left(\boldsymbol{\epsilon}_{\sigma}^{*} \cdot \boldsymbol{M}_{f i}\right)
$$

where

$$
\boldsymbol{M}_{f i}=\int d^{3} \boldsymbol{r} \psi_{f}^{*}(\boldsymbol{r})\left(\widetilde{\boldsymbol{\alpha}}_{(1)} e^{-\frac{i}{2} \boldsymbol{k} \boldsymbol{r}}-\widetilde{\boldsymbol{\alpha}}_{(2)} e^{\frac{i}{2} \boldsymbol{k} \boldsymbol{r}}\right) \psi_{i}(\boldsymbol{r})
$$


Table 5. Values in $\mathrm{keV}$ of the theoretical and experimental widths of radiative decays of $\Upsilon(3 s), \chi_{b 2}(2 p), \chi_{b 1}(2 p), \chi_{b 0}(2 p), \Upsilon(2 s)$.

\begin{tabular}{lrr}
\hline Decay & Theoretical & Experimental \\
\hline$\Upsilon(3 s) \rightarrow \gamma \chi_{b 2}(2 p)$ & 3.51 & $2.70 \pm 0.57$ \\
$\Upsilon(3 s) \rightarrow \gamma \chi_{b 1}(2 p)$ & 2.85 & $2.58 \pm 0.48$ \\
$\Upsilon(3 s) \rightarrow \gamma \chi_{b 0}(2 p)$ & 1.52 & $1.21 \pm 0.23$ \\
$\Upsilon(3 s) \rightarrow \gamma \eta_{b}(2 s)$ & 0.006 & $<0.013$ \\
$\Upsilon(3 s) \rightarrow \gamma \chi_{b 2}(1 p)$ & 0.149 & $0.204 \pm 0.045$ \\
$\Upsilon(3 s) \rightarrow \gamma \chi_{b 1}(1 p)$ & 0.036 & $0.019 \pm 0.012$ \\
$\Upsilon(3 s) \rightarrow \gamma \chi_{b 0}(1 p)$ & 0.032 & $0.056 \pm 0.013$ \\
$\Upsilon(3 s) \rightarrow \gamma \eta_{b}(1 s)$ & 0.009 & $0.011 \pm 0.003$ \\
\hline$\Upsilon(2 s) \rightarrow \gamma \chi_{b 2}(1 p)$ & 2.13 & $2.30 \pm 0.20$ \\
$\Upsilon(2 s) \rightarrow \gamma \chi_{b 1}(1 p)$ & 1.73 & $2.22 \pm 0.21$ \\
$\Upsilon(2 s) \rightarrow \gamma \chi_{b 0}(1 p)$ & 0.87 & $1.22 \pm 0.15$ \\
$\Upsilon(2 s) \rightarrow \gamma \eta_{b}(1 s)$ & 0.013 & $0.013 \pm 0.04$ \\
\hline$\chi_{b 2}(2 p) \rightarrow \gamma \Upsilon(2 s)$ & 18.77 & $15.10 \pm 5.60$ \\
$\chi_{b 2}(2 p) \rightarrow \gamma \Upsilon(1 s)$ & 10.27 & $9.80 \pm 2.30$ \\
\hline$\chi_{b 1}(2 p) \rightarrow \gamma \Upsilon(2 s)$ & 16.80 & $14.40 \pm 5.00$ \\
$\chi_{b 1}(2 p) \rightarrow \gamma \Upsilon(1 s)$ & 7.68 & $8.96 \pm 2.24$ \\
\hline$\chi_{b 0}(2 p) \rightarrow \gamma \Upsilon(2 s)$ & 11.77 & - \\
$\chi_{b 0}(2 p) \rightarrow \gamma \Upsilon(1 s)$ & 1.49 & - \\
\hline$\chi_{b 2}(1 p) \rightarrow \gamma \Upsilon(1 s)$ & 33.73 & - \\
\hline$\chi_{b 1}(1 p) \rightarrow \gamma \Upsilon(1 s)$ & 29.48 & - \\
\hline$\chi_{b 0}(1 p) \rightarrow \gamma \Upsilon(1 s)$ & 19.65 & - \\
\hline
\end{tabular}

has been calculated keeping the complete exponentials without the dipole approximation. The final formula for the transition rate reads therefore

$$
w=\frac{4}{3} \frac{e_{b}^{2}}{\hbar c} \omega_{f i} \Lambda_{f i}^{2} \sum \frac{\left|\boldsymbol{\epsilon}_{\sigma}^{*} \cdot \boldsymbol{M}_{f i}\right|^{2}}{2 j_{i}+1}
$$

A comment on the wave functions is in order. Due to the structure of $S_{f i}$ if we use the Breit corrected value for the transition frequency, namely the physical one, it seems reasonable to take the corresponding spinors at the lowest perturbation order. From Table 4, however, we see that the states with $j=0, \eta_{b}$ and $\chi_{b 0}$, have a considerably larger hyperfine shift than the other states, maximal in their respective multiplets. These $j=0$ are related by a parity transformation and in our model they are determined by a second order differential system instead of by a fourth order one. The inclusion of the first order corrections in their wave functions makes a really great improvement on the results of the decay transition rate. Still in the context of radiative meson decays, an analogous situation was met in [12] for the relativistic corrections in $v^{4} / c^{4}$. We shall adopt here the same solution suggested in that paper, namely we assume unperturbed wave functions for all the $j \neq 0$ states and wave functions corrected at the first order for all the $j=0$ states.

In Table 5 we show our numerical results for the measured decay widths, comparing them with the experimental values. In Table 6 we present some further predictions for not yet measured transitions, comparing them with theoretical results obtained by different methods. 
Table 6. Comparison of the previsions for the theoretical widths of some radiative decays of $\chi_{b 2}, h_{b}, \chi_{b 1}, \chi_{b 0}$ and $\Upsilon$. Units are $\mathrm{eV}$. ${ }^{*}$ This value is in agreement with the value (3.6 \pm 2.9$) \mathrm{eV}$ of [11].

\begin{tabular}{lrrr}
\hline \multicolumn{1}{c}{ Decay } & Ours & Ref[8] & Ref[10] \\
\hline$h_{b}(2 p) \rightarrow \gamma \eta_{b}(2 s)$ & 20681 & 17600 & 16600 \\
$h_{b}(2 p) \rightarrow \gamma \eta_{b}(1 s)$ & 16884 & 14900 & 17500 \\
\hline$\Upsilon(2 s) \rightarrow \gamma \eta_{b}(2 s)$ & 0.369 & 0.58 & 0.59 \\
\hline$\eta_{b}(2 s) \rightarrow \gamma \Upsilon(1 s)$ & 65.41 & 45 & 64 \\
\hline$\chi_{b 2}(1 p) \rightarrow \gamma h_{b}(1 p)$ & 0.015 & 0.089 & \\
$\chi_{b 2}(1 p) \rightarrow \gamma \Upsilon(1 s)$ & 33731 & 39150 & 31800 \\
\hline$h_{b}(1 p) \rightarrow \gamma \chi_{b 1}(1 p)$ & 0.050 & 0.012 & 0.0094 \\
$h_{b}(1 p) \rightarrow \gamma \chi_{b 0}(1 p)$ & 0.124 & 0.86 & 0.90 \\
$h_{b}(1 p) \rightarrow \gamma \eta_{b}(1 s)$ & 39318 & 43660 & 35800 \\
\hline$\Upsilon(1 s) \rightarrow \gamma \eta_{b}(1 s)$ & $3.101^{*}$ & 9.34 & 10 \\
\hline
\end{tabular}

\section{References}

[1] R. Giachetti, E. Sorace, J. Phys. A, 38, 1345 (2005).

[2] R. Giachetti, E. Sorace, J. Phys. A, 39, 15207 (2006).

[3] R. Giachetti, E. Sorace, Phys. Rev. D, 87, 034021 (2013).

[4] A. Barducci, R. Giachetti, E. Sorace, J. Phys. B: At. Mol. Opt. Phys. 48, 085002 (2015).

[5] R. Giachetti, E. Sorace, Phys. Rev. Lett., 101, 190401 (2008).

[6] A. Barducci, R. Giachetti, E. Sorace, arXiv:1604.08043, (2016).

[7] Review of Particle Physics, Particle Data Group, Chinese Physics C, 38, Number 9, (2014).

[8] W.-J. Deng, H. Liu, L.-C. Gui, X.-H. Zhong, arXiv 1607.05093 (2016).

[9] In [3] the influence of the Breit correction is extensively discussed and it is shown that its effect becomes larger and larger for decreasing meson masses. Notice also that since the publication of that paper, in addition to the value $9974.0 \pm 4.4 \mathrm{MeV}$ for the mass of $\eta_{b}(2 s)$ determined by the CLEO collaboration and in perfect agreement with our calculation (see S. Dobbs, Z. Metreveli, A. Tomaradze, T. Xiao, and Kamal K. Seth, Phys. Rev. Lett. 109, 082001 (2012) and references therein), the value of $9999.0 \pm 6.3 \mathrm{MeV}$ has been proposed by the BELLE collaboration (see S. Sandilya et al., Phys. Rev. Lett. 111, 112001 (2013)).

[10] J. Segovia, P.G. Ortega, D.R. Entem, F. Fernandez, Phys. Rev. D, 93, 074027 (2016).

[11] N. Brambilla, P. Pietrulewicz, A. Vairo Phys. Rev. D 85, 094005 (2012).

[12] H. Grotch, D.A. Owen, K.J. Sebastian, Phys. Rev. D 30, 1924 (1984). 